\title{
DECOMPOSITIONS OF GENERALIZED COMPLETE GRAPHS
}

\author{
BENJAMIN R. SMITH
}

(Received 30 June 2009)

2000 Mathematics subject classification: primary 05C70; secondary 05C38, $05 \mathrm{C} 15$. Keywords and phrases: graph decomposition, $k$-cycle, generalized complete graph.

A decomposition of a graph $G$ is a collection of edge-disjoint subgraphs of $G$ whose edges partition the edges of $G$. In the case where each of these subgraphs is isomorphic to some graph $H$, we say that $G$ admits a decomposition into $H$. The problem of determining whether a given graph $G$ admits a decomposition into a given graph $H$ is the primary focus of this thesis. In particular, we are concerned with those cases in which $H$ is a cycle.

Obvious necessary conditions for a graph $G$ (having nonempty edge set) to admit a decomposition into cycles of length $k$ are that: (i) $G$ contains at least $k$ vertices; (ii) every vertex in $G$ has even degree; and (iii) the total number of edges in $G$ is a multiple of the cycle length $k$. Recent papers by Alspach and Gavlas [1] and Šajna [6] have shown that these three necessary conditions are sufficient in the case where $G$ is either $K_{2 n+1}$ (the complete graph of odd order), or $K_{2 n}-F$ (the complete graph of even order minus a 1-factor). In this thesis we examine whether conditions (i), (ii) and (iii) above are also sufficient when $G$ is one of $\lambda K_{n}$ (the $\lambda$-fold complete multigraph), $K_{n} * \bar{K}_{m}$ (the complete equipartite graph having $n$ parts of size $m$ ) or $\lambda K_{n} * \bar{K}_{m}$ (the $\lambda$-fold complete equipartite graph).

In Chapter 3 we consider $k$-cycle decompositions of $\lambda K_{n}$. We also consider directed cycle decompositions of $\lambda K_{n}^{*}$, which is the graph obtained by replacing each edge in $\lambda K_{n}$ with a pair of oppositely directed arcs. We give various new cyclic and 1-rotational decompositions of these graphs, in particular for those cases in which the cycle length $k$ is a factor of the multiplicity $\lambda$. Ultimately we establish necessary and sufficient conditions for the existence of a $k$-cycle decomposition of $\lambda K_{n}$, and of a directed $k$-cycle decomposition of $\lambda K_{n}^{*}$, in the cases where $k$ is an odd prime.

Thesis submitted to the University of Queensland, November 2008. Degree approved, April 2009. Supervisor: Associate Professor Elizabeth J. Billington.

(C) 2009 Australian Mathematical Publishing Association Inc. 0004-9727/2009 \$16.00 
In Chapters 4, 5 and 6 we turn our attention to $k$-cycle decompositions of $K_{n} * \bar{K}_{m}$. In [5], Manikandan and Paulraja established necessary and sufficient conditions for the existence of such decompositions in the case where $k$ is an odd prime. In Chapter 4, using new techniques, we extend this result to include cases in which $k$ is either twice an odd prime or three times an odd prime. In Chapter 5 we consider complete equipartite graphs having a 'small' number of parts; in particular, we establish necessary and sufficient conditions for decomposing $K_{n} * \bar{K}_{m}$ into $k$-cycles in the cases where $n=4,5$ (the cases $n=2$ and $n=3$ having already been dealt with by Sotteau [7] and Cavenagh [3], respectively). We also consider $k$-path decompositions in these cases. In Chapter 6 we present a new technique-using edge labelled decompositions of multigraphs-for obtaining $k$-cycle decompositions of $K_{n} * \bar{K}_{m}$ in cases where $k$ is a factor of $m^{2}$. We use this technique to establish necessary and sufficient conditions for $K_{n} * \bar{K}_{m}$ to admit a decomposition into cycles of length $p^{2}$ where $p$ is an odd prime.

In Chapter 7 we examine so called gregarious $k$-cycle decompositions of $K_{n} * \bar{K}_{m}$; that is, those in which each cycle in the decomposition has each of its vertices lying in a different partite set of $K_{n} * \bar{K}_{m}$. We prove that the well-known necessary conditions for the existence of such a decomposition are sufficient for all $n, m$ and even $k$ if and only if they are sufficient for all $n$ in the range $k \leq n<3 k$ with $m$ odd, and all $n$ in the range $k \leq n<2 k$ with $m$ even. We then use this fact to deal with cases in which $k=6,8$. Using several new construction methods, we also establish necessary and sufficient conditions for $K_{n} * \bar{K}_{m}$ to admit a decomposition into gregarious cycles of length $p$ where $p$ is an odd prime.

Chapter 8 gives some generalizations of the results in earlier chapters to $k$-cycle decompositions of $\lambda K_{n} * \bar{K}_{m}$, the $\lambda$-fold complete equipartite graph. Necessary and sufficient conditions for the existence of such decompositions have been established in the case $k=3$ by Hanani [4], and in the case $k=5$ by Billington et al. [2]. In this chapter we extend these results to include all prime values of $k$.

Finally, in Chapter 9 we establish necessary and sufficient conditions for decomposing complete equipartite graphs into closed trails of length $k$.

\section{References}

[1] B. Alspach and H. Gavlas, 'Cycle decompositions of $K_{n}$ and $K_{n}-I$ ', J. Combin. Theory Ser. B 81 (2001), 77-99.

[2] E. J. Billington, D. G. Hoffman and B. M. Maenhaut, 'Group divisible pentagon systems', Util. Math. 55 (1999), 211-219.

[3] N. J. Cavenagh, 'Decompositions of complete tripartite graphs into k-cycles', Australas. J. Combin. 18 (1998), 193-200.

[4] H. Hanani, 'Balanced incomplete block designs and related designs', Discrete Math. 11 (1975), 255-369.

[5] R. S. Manikandan and P. Paulraja, ' $C_{p}$-decompositions of some regular graphs', Discrete Math. 306 (2006), 429-451.

[6] M. Šajna, 'Cycle decompositions III: complete graphs and fixed length cycles', J. Combin. Des. 10 (2002), 27-78. 
[7] D. Sotteau, 'Decomposition of $K_{m, n}\left(K_{m, n}^{*}\right)$ into cycles (circuits) of length $2 k$ ', J. Combin. Theory Ser. B 30 (1981), 75-81.

BENJAMIN R. SMITH, School of Mathematics and Physics, The University of Queensland, Brisbane 4072, Australia e-mail: bsmith.maths@gmail.com 